\title{
Inhibition of ERCC5 Attenuates Proliferation and Invasion while Promotes Apoptosis in Ovarian Cancer
}

\author{
LI JIANG, XINHUA BU*, HUA QIAN, DONGLAN YUAN, SHAOJUN WU AND YINLING ZHAO \\ Department of Obstetrics and Gynecology, Jiangsu Taizhou People's Hospital, the Fifth Affiliated Hospital of Nantong \\ University, Taizhou, Jiangsu 225300, P. R. China
}

Jiang et al.: Inhibition of ERCC5 attenuates proliferation and invasion

\begin{abstract}
The present investigation dealt with differential expression of ERCC5 in different ovarian cancer cells and the potential mechanism involved. By detecting the expression in both SKOV3 and Caov4 cell lines, it was found that the expression of ERCC5 in SKOV3 was significantly higher than that in Caov4. An SKOV3 cell line with low ERCC5 expression by ribonucleic acid interference and Caov4 cell line with high ERCC5 expression by transient transfection was constructed to compare the effect of ERCC5 abnormal expression on the biological behaviour of ovarian cancer cells. The results showed that high expression of ERCC5 could significantly promote proliferation and invasion of ovarian cancer but inhibit apoptosis, while low expression of ERCC5 could significantly promote apoptosis and inhibit proliferation and invasion. The effect of ERCC5 abnormal expression on potential regulation pathways was studied using Western blot. It was found that ERCC5 abnormal expression could regulate the Wnt pathway. It was demonstrated that inhibition of ERCC5 expression could attenuate proliferation and invasion while promote apoptosis of ovarian cancer cells by suppressing the Wnt pathway.
\end{abstract}

Key words: ERCC5, ovarian cancer, biological behaviour, Wnt pathway

Epithelial ovarian cancer (referred to as ovarian cancer, OC) is one of the most common gynaecological malignant tumours in women with high mortality, rapid transfer, strong invasion ability and high recurrence rate. Five-year survival rate is less than $30 \%$ in advanced ovarian cancer patients. Because there is no specific symptom of early ovarian cancer and the lack of highly specific and sensitive diagnostic methods in the clinic, more than $70 \%$ of patients are in advanced stage when diagnosed. The maintenance of DNA repair capability (DRC) depends on sufficient expression of DNA repair gene and the lack of expression could not activate the repair pathway, resulting in a decrease of DRC and increased tumour susceptibility ${ }^{[1,2]}$. Nucleotide excision repair (NER) is the most important DNA repair pathway that can repair DNA damage in a broad spectrum, including UVinduced damage, DNA adducts and some other kinds of oxidative damage and at least 30 kinds of proteins were involved ${ }^{[3,4]}$. ERCC5, known as the XPG gene, is one of the eight key genes of the NER pathway ${ }^{[5]}$. There are 5 main DNA repair pathways in the human body. Among them, NER pathway mainly repairs endogenous and exogenous DNA damage affecting regional chromosome structure to maintain the stability of DNA, and its

*Address for correspondence E-mail: m16531756472_1@163.com genetic alteration will affect DNA repair ability, resulting in abnormal DNA replication and abnormal cell proliferation and differentiation ${ }^{[6]}$. Therefore, the occurrence of many tumours is closely related to the changes of gene expression and the deletion or downregulation of gene function in NER pathway. The effect of the ERCC5 gene is to recognize and cleave the 3' end of the damaged DNA strand in the NER pathway and to function as a non-enzymatic role for ERCC1 gene which is responsible for cleaving the 5' end in the NER pathway ${ }^{[5]}$. Many studies have found that the abnormal expression of NER pathway genes plays an important role in the development and prognosis of many kinds of tumours. For example, Zhao et al. found that elevated expression of ERCC6 in colorectal cancer can cause resistance to 5-fluorouracil and was related to poor prognosis ${ }^{[7]}$. Jeong et al. found that the survival time of non-small cell lung cancer patients with ERCC 1 high expression was significantly shortened ${ }^{[8]}$. As an important member of the NER pathway gene, ERCC5 has also been found to be abnormally expressed in a variety of tumours, including OC. Walsh et al. found that ERCC5 was abnormally expressed in ovarian cancer tissues, and the survival of patients with low expression of ERCC5 was significantly prolonged ${ }^{[9]}$. However, the impact of ERCC5 abnormal expression on the biological behaviour of $\mathrm{OC}$ and the potential mechanism has not yet been reported.OC cell lines 
SKOV3 was selected in this study. Real-time PCR and western blot were carried out to compare the expression of ERCC5 in SKOV3 cell line. The effect of low or overexpression of ERCC5 on the behaviour of OC cells was observed using real-time PCR and Western blot. Finally, The effect of inhibition of ERCC5 expression on Wnt pathway was studied using Western blot. O C cell lines SKOV3, OVCA4, Cov434 and Caov3 were purchased from the cell bank of Chinese Academy of Sciences (Shanghai, China). ERCC5 silence cell line (siRNA-ERCC5) and overexpression cell line (ERCC5) were all constructed by transient transfection with lipo2000 according to the manufacturer's specification. Interference RNA was purchased from Guangzhou Geneseed Company and plasmid was purchased from Shanghai Genechem Company. SKOV3 was cultured with McCoys 5A medium supplemented with $10 \%$ fetal bovine serum (FBS, Thermo Fisher), while the other cell lines were cultured with L15 medium supplemented with $20 \%$ FBS (Hyclon). The cell incubator was set to $5 \%$ carbon dioxide and the temperature was set at $37^{\circ}$. OC cells were inoculated in to 24-well plate treated with collagen and cultured in an incubator for $24 \mathrm{~h}$. After discarding the culture medium and washing with PBS for 3 times, the cells were fixed with a fixing solution for $10 \mathrm{~min}$. Then the cells were stained and counteduisng microscopic photography. Trizol buffer was used to extract total RNA (KeyGEN, Nanjing, China). RNA was reverse transcribed to cDNA by PrimeScript RT Master Mix (Takara). RT-qPCR was performed on Eppendorf equipment using SYBR Premix Ex Taq (Takara, Liaoning, China) according to the manufacturer's protocols. RIPA buffer was used to extract total proteins (Aidlab Biotechnologies, Beijing,
China). Tissue lysates were centrifuged at $12000 \mathrm{rpm}$ for $30 \mathrm{~min}$ at $4^{\circ}$. Supernatant was collected and protein was quantified with a BCA reagent kit. Lysates were boiled at $100^{\circ}$ for $5 \mathrm{~min}$ and $40 \mathrm{~g}$ of total protein was separated by $4-12 \%$ SDS-PAGE and then transferring to polyvinylidenedifluoride (PVDF) membranes. The PVDF membranes were blocked with non-fat milk for $1 \mathrm{~h}$ and then incubated with primary antibodies at $4^{\circ}$ overnight. The membranes were then incubated with secondary antibodies for $2 \mathrm{~h}$ at room temperature. Chemiluminescence reagent ECL Plus was used to visualize the bands and the results were analysed by Image J software.Statistical analyses were performed with SPSS18.0 software (Chicago, USA). Comparison of gene differential expression between SKOV3NC, Caov4NC and SKOV3KD, Caov4OE was performed by student's $\mathrm{T}$ test. Two-tailed $\mathrm{P}$ values $<0.05$ was considered statistically significant.RT-qPCR analysis showed that the expression of ERCC5 in OC tissues was significantly higher than that in normal tissues (fig. 1A). In addition, the production of ERCC5 in OC cell lines was also markedly higher than that in normal ovarian cells (fig. 1B). ERCC5 expression was found to be the highest in OC cell lines, hence SKOV3 was chosen as the OC cell model in the following in vitro experiments. To investigate the effect of ERCC5 on SKOV3 apoptosis, Western blot analysis was performed in this study. Firstly, siRNA-ERCC5 was used to knock down the expression of ERCC5 (fig. 2A and 2B). Then, Western blot analysis was performed to detect the apoptotic proteins of Caspase 3 and Bax, the results showed that inhibition of ERCC5 significantly promoted SKOV3 cell apoptosis (fig. 2C and 2D). Contrary to the effect of low ERCC5 expression on
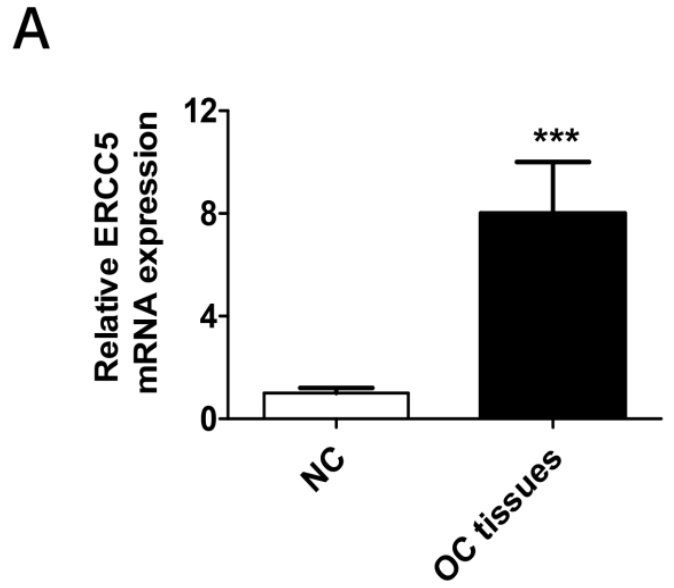

B

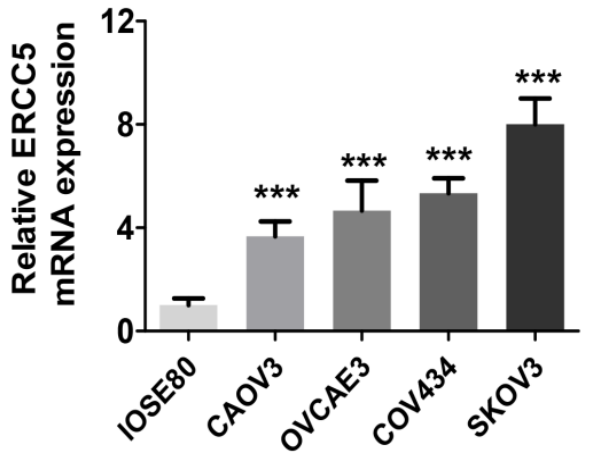

Fig. 1: Upregulation of ERCC5 expression in $\mathrm{OC}$ tissues and $\mathrm{OC}$ cell lines

A-B. RT-qPCR analysis to examine the mRNA expression of ERCC5 in OC tissues and OC cell lines. All experiments were performed in triplicate. ${ }^{*} \mathbf{p}<0.05,{ }^{* *} \mathbf{p}<0.01,{ }^{* * *} \mathbf{p}<0.001$ 
SKOV3 apoptosis, inhibition of ERCC5 can attenuate the OC cell proliferation and invasion. It was found that low ERCC5 expression could significantly inhibit the proliferation and invasion of SKOV3 cells (fig 3A-3C). To analyse the effect of upregulation of ERCC 5 in OC, adenovirus was used to overexpress the ERCC5 (fig. 4A and 4B). Western blot analysis showed that ERCC5 can significantly increase the cleaved caspase 3 and Bax expression in SKOV3 cell line (fig. 4C-4E). MTT assay results showed that overexpression of ERCC5 significantly elevated the level of OC cell proliferation (fig. 5A). Transwell assay confirmed that overexpression of ERCC5 further promote the invasion of SKOV3 cell (fig. 5B-5C). Node proteins including
P38 (phosphorylation P38), JNK (phosphorylation JNK) and ERK (phosphorylation ERK) of MAPK pathway, the node protein Ik-B $\alpha$ of NF-kB pathway and the node protein $\beta$-catenin of Wnt pathway were chosen for further study. Western blot results showed that Knock-down of ERCC5 expression did not affect the expression of P38 (phosphorylated P38), JNK (phosphorylated JNK), IkB- $\alpha$ and ERK (phosphorylated ERK), but can significantly decrease the $\beta$-catenin expression (fig. 6A and 6B).DNA is the most important genetic material in life activities. Due to its structural specificity, DNA is vulnerable to attack by various intrinsic and exogenous physicochemical factors, causing genomic instability, while genomic instability
A

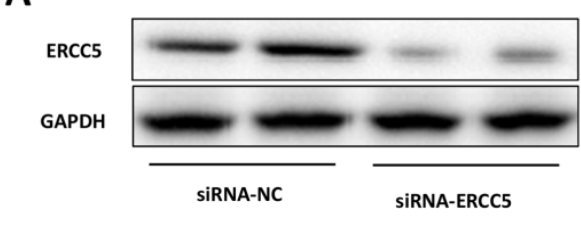

C

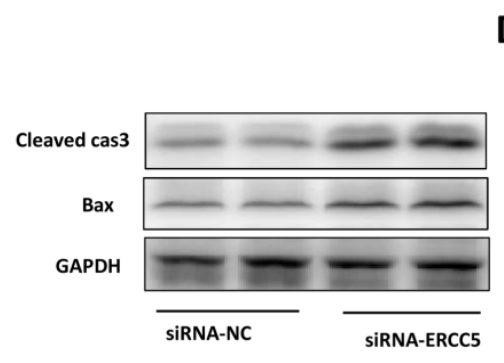

B
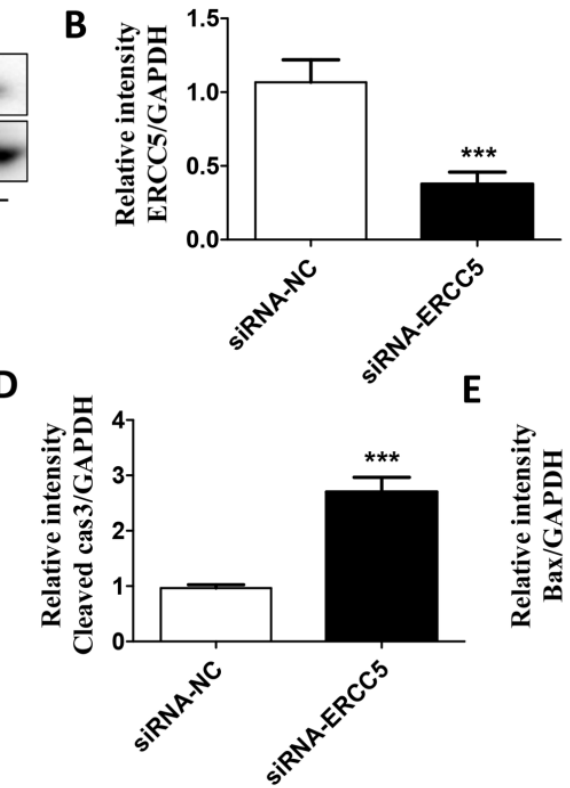

E

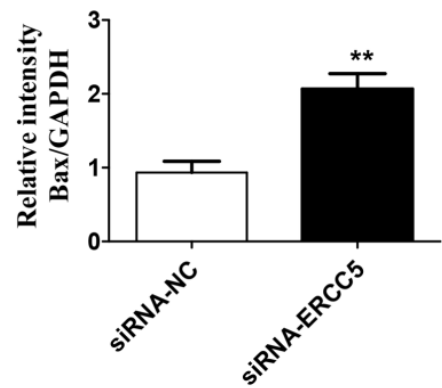

Fig. 2: Inhibition of ERCC5 promotes SKOV3 cell apoptosis

A. Western blot analysis to confirm the transfection efficiency of siRNA-ERCC5. B. Western blot analysis to detect apoptosis protein. All experiments were performed in triplicate. ${ }^{*} p<0.05, * * p<0.01, * * * p<0.001$

A

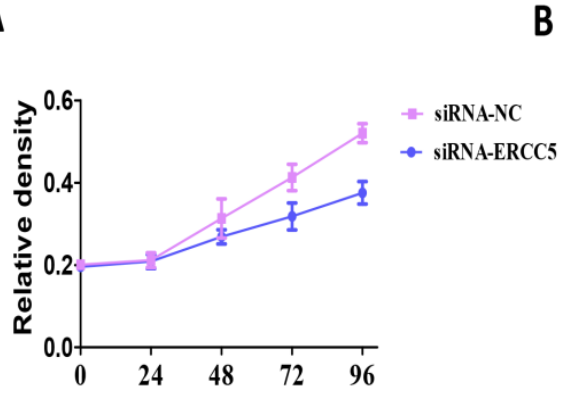

B

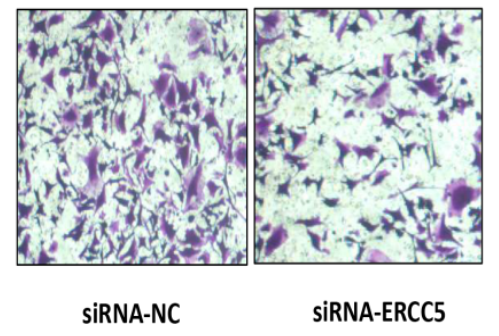

C

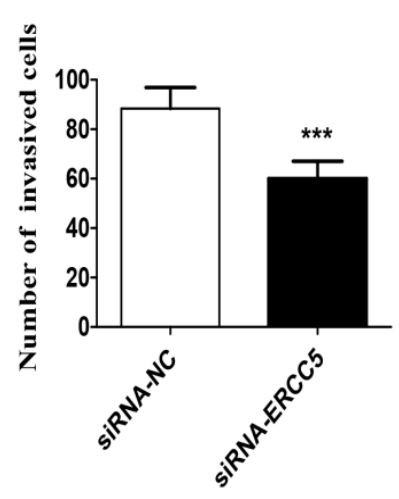

Fig. 3: Suppression of ERCC5 attenuates OC cells proliferation and invasion

A. MTT assay to detect the OC cell proliferation. B-C.Transwell was used to examine the invasion of OC cells. All experiments were performed in triplicate. ${ }^{*} \mathbf{p}<0.05, * * \mathbf{p}<0.01, * * * \mathbf{p}<0.001$ 
A

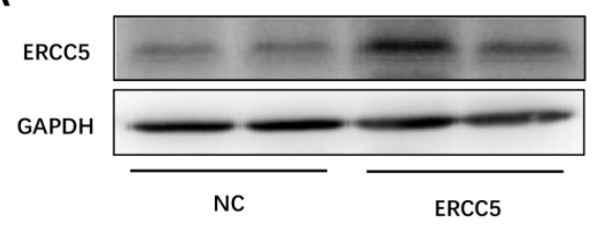

B

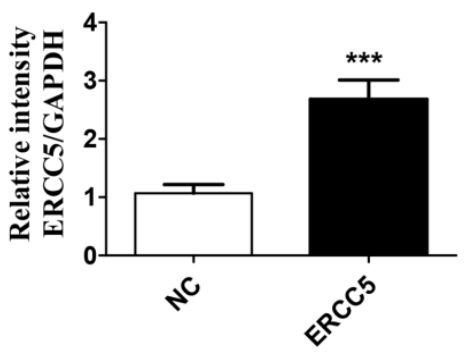

C

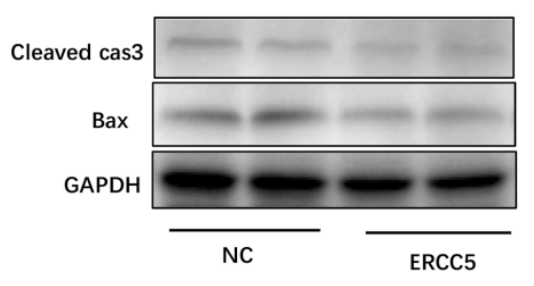

D

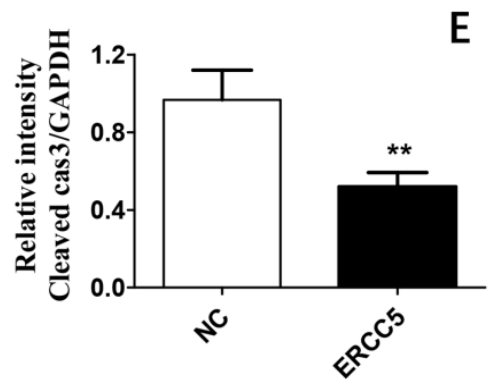

$\mathrm{E}$

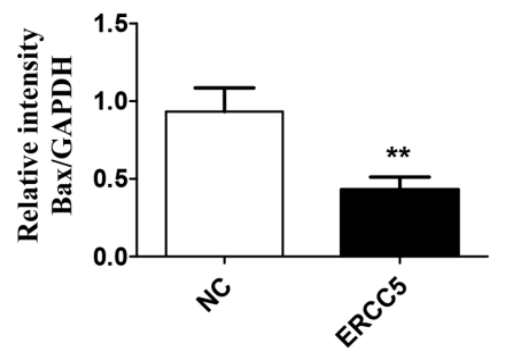

Fig. 4: Overexpression of ERCC5 can significantly promote $\mathrm{OC}$ cell apoptosis A. Western blot analysis to confirm the transfection efficiency of ERCC5 adenovirus. B. Western blot analysis to detect the apoptosis proteins. All experiments were performed in triplicate. ${ }^{*} \mathbf{p}<0.05,{ }^{* *} \mathbf{p}<0.01, * * * \mathbf{p}<0.001$

A

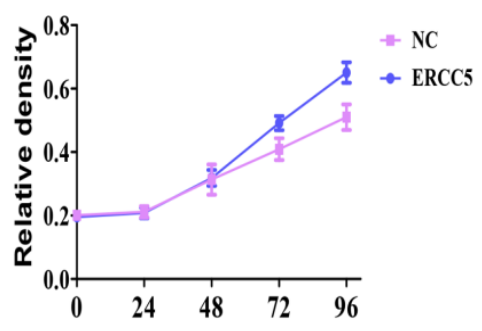

B

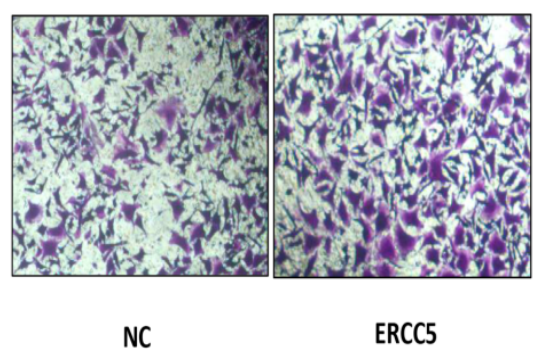

C

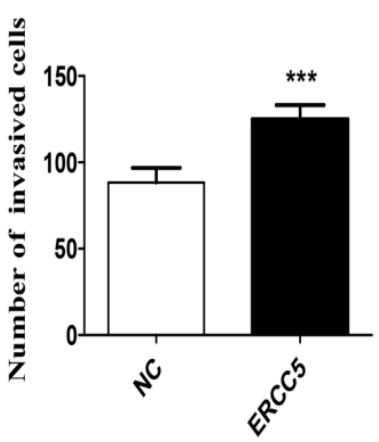

Fig. 5: Over expression of ERCC5 promote OC cell proliferation and invasion

A. MTT assay to detect the OC cell proliferation. B-C.Transwell was used to examine the invasion of OC cells. All experiments were performed in triplicate.. ${ }^{*} \mathbf{p}<0.05,{ }^{* *} \mathbf{p}<0.01,{ }^{* * *} \mathbf{p}<0.001$

is a tumour susceptibility root cause. DNA repair process is the most important part of the identification of DNA damage. Although there are many repair DNA damage mechanisms in human body, NER is the most important DNA damage repair system. ERCC5 (Excision repair cross complementation group 5), also known as XPG (Xeroderma pigmentosum complementary group $\mathrm{G})^{[10]}$, belongs to the RAD2/XPG family and is one of the key factors of NER repair pathway. It is located on chromosome 13q33, and is a kind of specific nuclease which is responsible for the
3' endonuclease in mammalian nucleic acid excision repair and is also involved in the $\mathrm{xPF} / \mathrm{ERCCl}$ complexmediated 5' endogenesis ${ }^{[11-13]}$. Studies have shown that ERCC5 gene is expressed in a variety of tumour tissues and cell lines and its expression level is related to the sensitivity of platinum drugs ${ }^{[9,14-18]}$.Many studies have found that ERCC5 gene played an important role in the development and prognosis of many kinds of tumours ${ }^{[19,20]}$. However, the relationship between ERCC5 gene and the development of ovarian cancer is rarely reported at present. In this study, two ovarian 
A

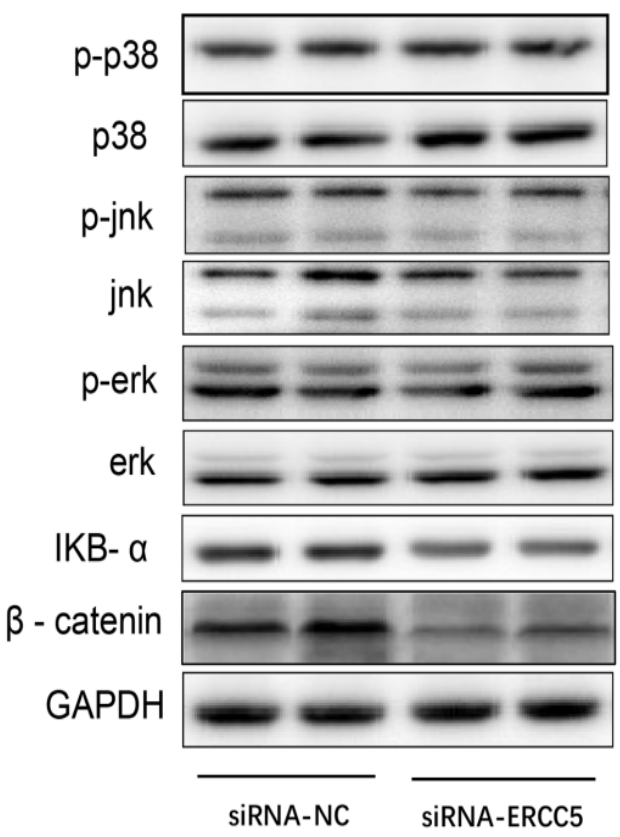

B

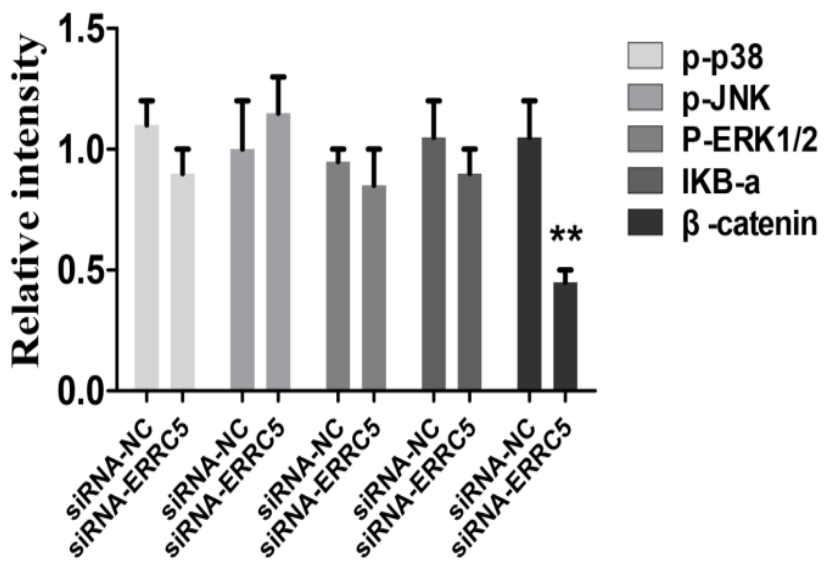

Fig. 6: Knock-down of ERCC5 expression in OC cells can activate Wnt pathway

A. Western blot analysis was used to explore the potential pathways of ERCC5 in OC cells. B. All the experiments were repeated three times independently. ${ }^{*} \mathbf{p}<0.05, * * \mathbf{p}<0.01, * * * \mathbf{p}<0.001$

cancer cell lines were selected. Cell lines with ERCC5 low expression and high expression were constructed by RNAi and plasmid transfection. Cell proliferation, apoptosis and invasion were assessed by RT-PCR and western blot to detect the expression of relevant indicators. The results showed that overexpression of ERCC5 could significantly promote the proliferation and invasion of ovarian cancer cells and inhibit the apoptosis of ovarian cancer cells. However, the low expression of ERCC5 could significantly inhibit the proliferation and invasion of ovarian cancer cells and promote their apoptosis. We further selected the node proteins of 3 classical signalling pathways including MAPK, Wnt and NF-kB pathway for Western blot detection and found that inhibition of ERCC5 expression did not affect the activity of MAPK and NF-kB pathway, but can significantly suppress the Wnt pathway, thereby affecting ovarian cancer biological behaviour of cells. In this study, the role of ERCC5 gene in the development and progression of ovarian cancer and its mechanism was primarily explored by in vitro cytology experiments, in order to provide a new theoretical basis for the prevention and treatment of ovarian cancer.

\section{Conflict of interest:}

All authors report no conflicts of interest in this work.

\section{REFERENCES}

1. Lochhead P, Emad M. El-Omar. Gastric cancer. Br Med Bull 2008;85:87-100.

2. Friedberg EC. How nucleotide excision repair protects against cancer. Nat Rev Cancer 2001;1:22-33.

3. Lehmann AR. DNA repair-deficient diseases, xeroderma pigmentosum, Cockayne syndrome and trichothiodystrophy. Biochimie 2003;85:1101-11.

4. Liu J, He C, Xing C, Yuan Y. Nucleotide excision repair related gene polymorphisms and genetic susceptibility, chemotherapeutic sensitivity and prognosis of gastric cancer. Mutat Res 2014;765:11-21.

5. Snyder SH. A common mutational pattern in Cockayne syndrome patients from xeroderma pigmentosum group G: implications for a second XPG function. Proc Natl Acad Sci USA 2006;103:19606.

6. Wood RD, Mitchell M, Sgouros J, Lindahl T. Human DNA repair genes. Science 2001;291:1284-9.

7. Zhao Z, Zhang G, Li W. Elevated Expression of ERCC6 Confers Resistance to 5-Fluorouracil and Is Associated with Poor Patient Survival in Colorectal Cancer. DNA Cell Biology 2017;36:781-6.

8. Jeong YH, Lee CK, Jo K, Hwang SH, Cha J, Lee JW, et al. Correlation Analysis and Prognostic Impact of (18)F-FDG PET and Excision Repair Cross-Complementation Group 1 (ERCC-1) Expression in Non-Small Cell Lung Cancer. Nucl Med Mol Imaging 2015;49:108-14.

9. Walsh CS, Ogawa S, Karahashi H, Scoles DR, Pavelka JC, Tran H, et al. ERCC5 is a novel biomarker of ovarian cancer prognosis. J Clin Oncol 2008;26:2952-8.

10. Mudgett JS, MacInnes MA. Isolation of the functional human excision repair gene ERCC5 by intercosmid recombination. Genomics 1990;8:623-33. 
11. Aboussekhra A, Biggerstaff $M$, Shivji MK, Vilpo JA, Moncollin V, Podust VN, et al. Mammalian DNA nucleotide excision repair reconstituted with purified protein components. Cell 1995;80:859-68.

12. Mu D, Hsu DS, Sancar A. Reaction mechanism of human DNA repair excision nuclease. J Biol Chem 1996;271:8285-94.

13. Wakasugi M, Reardon JT, Sancar A. The non-catalytic function of XPG protein during dual incision in human nucleotide excision repair. J Biol Chem 1997;272:16030-4.

14. Kelland LR. Preclinical perspectives on platinum resistance. Drugs 2000;59:1-8.

15. Furuta T, Ueda T, Aune G, Sarasin A, Kraemer KH, Pommier Y. Transcription-coupled nucleotide excision repair as a determinant of cisplatin sensitivity of human cells. Cancer Res 2002;62:4899-902.

16. Stevens EV, Nishizuka S, Antony S, Reimers M, Varma S, Young L, et al. Predicting cisplatin and trabectedin drug sensitivity in ovarian and colon cancers. Mol Cancer Ther 2008;7:10-8.

17. Koeppel F, Poindessous V, Lazar V, Raymond E, Sarasin A, Larsen AK. Irofulven cytotoxicity depends on transcriptioncoupled nucleotide excision repair and is correlated with
XPG expression in solid tumour cells. Clin Cancer Res 2004;10:5604-13.

18. Hamilton SR. Targeted therapy of cancer: new roles for pathologists in colorectal cancer. Modern Pathol 2008;21:S2330.

19. Zhang C, Liao Z, Yu G, Huang W, Song X. Study on association between ERCC5 single nucleotide polymorphism and susceptibility to esophageal cancer. J BUON 2017;22:97984.

20. Yang LQ, Zhang Y, Sun HF. Investigation on ERCC5 genetic polymorphisms and the development of gastric cancer in a Chinese population. Genet Mol Res 2016;15.

This is an open access article distributed under the terms of the Creative Commons Attribution-NonCommercial-ShareAlike 3.0 License, which allows others to remix, tweak, and build upon the work non-commercially, as long as the author is credited and the new creations are licensed under the identical terms

This article was originally published in a special issue, "Biomedical Research in Healthcare Setting" Indian J Pharm Sci 2020:82(3)Spl issue5;97-102 